ARTICLE

https://doi.org/10.1038/s41467-019-13773-3

\title{
Enhanced control of self-doping in halide perovskites for improved thermoelectric performance
}

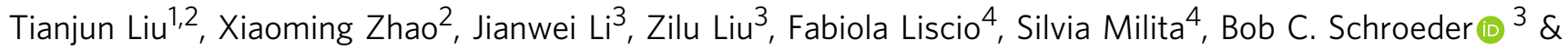
Oliver Fenwick ${ }^{1,2 \star}$

Metal halide perovskites have emerged as promising photovoltaic materials, but, despite ultralow thermal conductivity, progress on developing them for thermoelectrics has been limited. Here, we report the thermoelectric properties of all-inorganic tin based perovskites with enhanced air stability. Fine tuning the thermoelectric properties of the films is achieved by self-doping through the oxidation of tin (II) to tin (IV) in a thin surface-layer that transfers charge to the bulk. This separates the doping defects from the transport region, enabling enhanced electrical conductivity. We show that this arises due to a chlorine-rich surface layer that acts simultaneously as the source of free charges and a sacrificial layer protecting the bulk from oxidation. Moreover, we achieve a figure-of-merit (ZT) of $0.14 \pm 0.01$ when chlorine-doping and degree of the oxidation are optimised in tandem.

\footnotetext{
${ }^{1}$ School of Engineering and Material Sciences, Queen Mary University of London, Mile End Road, London E1 4NS, UK. ${ }^{2}$ The Organic Thermoelectrics Laboratory, Materials Research Institute, Queen Mary University of London, Mile End Road, London E1 4NS, UK. ${ }^{3}$ Department of Chemistry, University College London, 20 Gordon Street, London WC1H OAJ, UK. ${ }^{4}$ Istituto per la Microelettronica e Microsistemi (IMM)-Consiglio Nazionale delle Ricerche (CNR), Via Gobetti 101, 40129 Bologna, Italy. ${ }^{\star}$ email: o.fenwick@gmul.ac.uk
} 
W ith rapidly rising greenhouse gas emissions to the atmosphere, it is paramount to develop technologies able to generate energy at negligible cost to the environment, and to reverse the currently accelerating climatic changes. However, to successfully fulfil the transition from fossil fuels to renewable energy sources, we can no longer rely solely on existing materials, but must focus on the synthesis of other material classes with improved properties. Halide perovskites have been recognized as promising photovoltaic materials ${ }^{1-3}$ achieving a power conversion efficiency exceeding $25 \%{ }^{4}$, due to their large absorption coefficients, high charge carrier mobilities ${ }^{5}$ and large carrier diffusion lengths $s^{6}$. They are a highly versatile class of semiconductors, with a band gap that is tuneable through the composition of the inorganic framework, the choice of organic or inorganic cation, stoichiometry, and through selfassembly into layered structures ${ }^{7-10}$ and nanoparticles ${ }^{11}$. This diversity in structure has enabled the range of applications of these materials to extend to other optoelectronic devices, including light-emitting diodes (LEDs) ${ }^{12-14}$, X-ray detectors ${ }^{15,16}$ and lasers ${ }^{17,18}$

Despite intense research on halide perovskite materials for optoelectronics, there have only been a small number of experimental studies on their thermoelectric properties, where a temperature gradient across the material can move free charge carriers and generate a thermal voltage. Thermoelectric generators can produce electrical power from temperature gradients, and to do so efficiently, must use materials possessing a high figure-of-merit, ZT:

$$
\mathrm{ZT}=\sigma \alpha^{2} T / \kappa
$$

where $\sigma, \alpha$ and $\kappa$ are the electrical conductivity, Seebeck coefficient and thermal conductivity, respectively. $T$ is the temperature. Halide perovskites have an $\mathrm{ABX}_{3}$ stoichiometry comprising a network of inorganic (metal-halide) octahedra with loosely bound organic or inorganic cations occupying the cavities between octahedra. These cations provide rattling modes which scatter phonons, enabling ultralow values of thermal conductivity that are now well-documented ${ }^{19,20}$. Combined with the high charge mobilities ${ }^{5}$ observed in many halide perovskites, the relatively small number of experimental reports of ZT to date ${ }^{21,22}$ in these materials is perhaps surprising.

In 2014, He et al. studied thermoelectric properties of methylammonium lead iodide $\left(\mathrm{MAPbI}_{3}\right)$ and methylammonium tin iodide $\left(\mathrm{MASnI}_{3}\right)$ by ab initio calculations ${ }^{23}$. They found that both materials exhibit small carrier effective mass and weak phononphonon and hole-phonon couplings, and predicted ZT in the range of 1-2, in-line with state-of-the-art thermoelectric materials. Shortly afterwards, Mettan et al. ${ }^{21}$ measured the thermoelectric properties of $\mathrm{MAPbI}_{3}$ and $\mathrm{MASnI}_{3}$ bulk crystals ${ }^{21}$ finding that photo-induced doping of $\mathrm{MAPbI}_{3}$ and chemical doping of $\mathrm{MASnI}_{3}$ improved ZT. They concluded that $\mathrm{MAPbI}_{3}$ would be a good candidate for the thermoelectric applications due the high hole mobility, large Seebeck coefficient and a remarkably low thermal conductivity. However, the low charge carrier density is a barrier to further development. Low charge carrier density in these materials is a product of ionic compensation of charged point $\operatorname{defects}^{24}$, as well as a defect tolerant electronic structure arising from bonding orbitals at the conduction band minimum, and antibonding orbitals at the valence band maximum ${ }^{25}$. The resulting low density of deep defects is an excellent feature for optoelectronic applications since defects can quench electroluminescence in LEDs or lead to recombination of photo-generated charges in solar cells. On the other hand, thermoelectric applications require charge densities typical of heavily doped semiconductors $\sim 10^{18}-10^{20} \mathrm{~cm}^{-3}$, and doping would usually come from defect sites, such as substitution of a higher valency metal atom on the perovskite B-site ${ }^{26}$. This makes development of halide perovskites for thermoelectrics challenging.

An exception are the lead-free tin halide perovskites, such as the cubic perovskite $\mathrm{CH}_{3} \mathrm{NH}_{3} \mathrm{SnI}_{3}$, which shows metallic conductivity ${ }^{27}$. Takahashi et al. ${ }^{28}$ noted that high conductivity in $\mathrm{CH}_{3} \mathrm{NH}_{3} \mathrm{SnI}_{3}$ bulk crystals arises from a self-doping process through the oxidation of $\mathrm{Sn}^{2+}$ to $\mathrm{Sn}^{4+28}$. In 2017, Lee et al. reported the ultralow thermal conductivity of a single $\mathrm{CsSnI}_{3}$ nanowire and a ZT of 0.11 at $320 \mathrm{~K}^{22}$, whilst Saini et al. report a ZT in thin films of 0.137 at $292 \mathrm{~K}^{29}$. However, the underlying physical mechanisms that determine thermoelectric performance of halide perovskite materials are not completely understood, and significant issues remain unaddressed such as identification of ZT optimisation strategies.

In this work, we develop a series of vacuum thermal evaporation methods to fabricate lead-free $\mathrm{CsSnI}_{3}$ perovskite thin films. We find air stability and electrical conductivity of our films to be highly tuneable by the deposition process with films formed by sequential deposition of the precursors yielding electrical conductivity 25 times that of films formed by co-evaporation of the same precursors. Compared with organic-inorganic hybrid perovskites, allinorganic halide perovskites present significant improvements in thermal stability ${ }^{30,31}$, but we enhance this further by developing a method to substitutionally dope chlorine into the perovskite structure in the top $10 \mathrm{~nm}$ of our films. A by-product of air exposure is the oxidation of $\mathrm{Sn}^{2+}$ to $\mathrm{Sn}^{4+}$ (self-doping), and we exploit this in a controlled manner to fine tune the electrical conductivity and thermoelectric properties of the mixed halide $\mathrm{CsSnI}_{3-\mathrm{x}} \mathrm{Cl}_{\mathrm{x}}$ perovskite thin films. We quantify the Sn oxidation states as a function of depth in mixed halide perovskite films using Auger electron spectroscopy, showing an unusual mechanism whereby an oxidised top surface-layer $(<10-\mathrm{nm}$ thick) is responsible for electrical doping the underlying film (250-300-nm thick). In this surface doping configuration, the dopants do not disrupt the crystal structure in the part of the film responsible for charge transport. In fact our Seebeck measurements indicate that the electrical doping levels in our films rise in tandem with the amount of $\mathrm{Cl}$ substituted in the top layers, showing that chlorine doping is simultaneously providing free charges to the system and acting as a sacrificial surface layer that slows oxidation of the bulk. We furthermore verify the applicability of the Wiedemann-Franz law in this class of materials with a value of the Lorenz number close to the Sommerfeld value, and achieve a ZT of 0.14 at $345 \mathrm{~K}$ upon simultaneous optimisation of the degree of $\mathrm{Cl}$-doping and the degree of oxidation.

\section{Results}

Thermal vapour deposition of $\mathrm{CsSnI}_{3}$ perovskite films. Past approaches to synthesize $\mathrm{CsSnI}_{3}$ have included solution processing by spin-coating ${ }^{31}$ and growth of single crystals ${ }^{22,32}$. In our case we have developed thermal vapour deposition approaches in order to achieve a high quality of films with fine control over morphology and composition.

Starting from the precursors caesium iodide (CsI) and stannous iodide $\left(\mathrm{SnI}_{2}\right)$, we developed three different vacuum deposition methods to prepare the perovskite films: co-evaporation, sequential deposition and seed layer plus sequential deposition (SLS) (Fig. 1a-c). For the co-evaporation process (Fig. 1a), the perovskite was obtained directly from simultaneous vacuum thermal evaporation of the two precursor materials $\left(\mathrm{SnI}_{2}\right.$ and $\left.\mathrm{CsI}\right)$. For the sequential deposition method (Fig. 1b), CsI and $\mathrm{SnI}_{2}$ were sequentially deposited to form a bilayer film which was then baked to form the perovskite. For the SLS method (Fig. 1c), a coevaporated perovskite seed layer was introduced before sequential deposition, and the film was post-baked to form the perovskite 
a

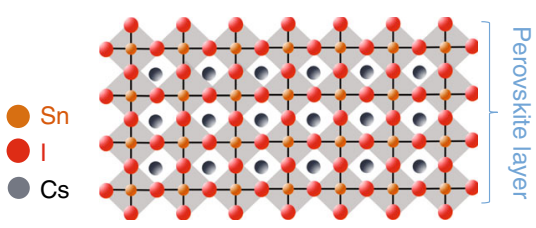

d

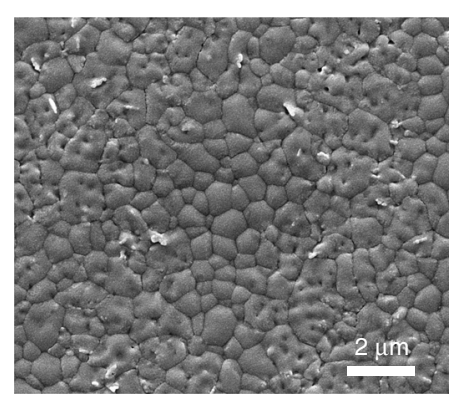

9

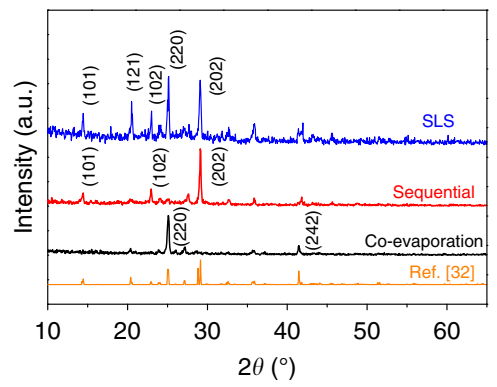

b

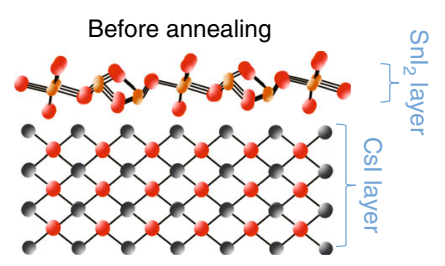

e

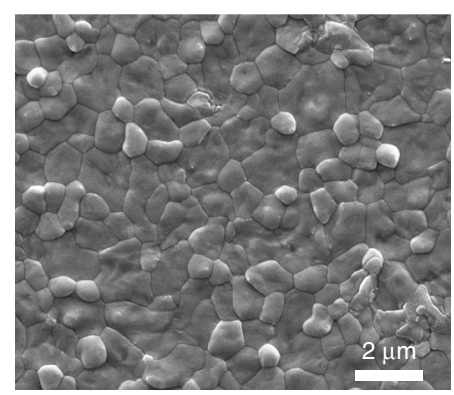

h

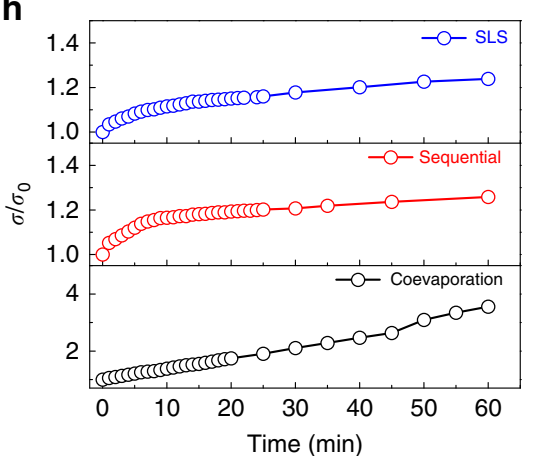

C

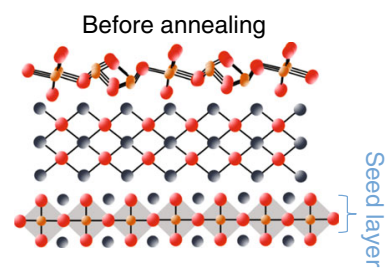

f

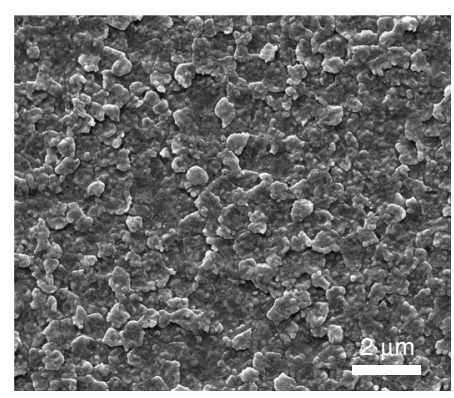

i

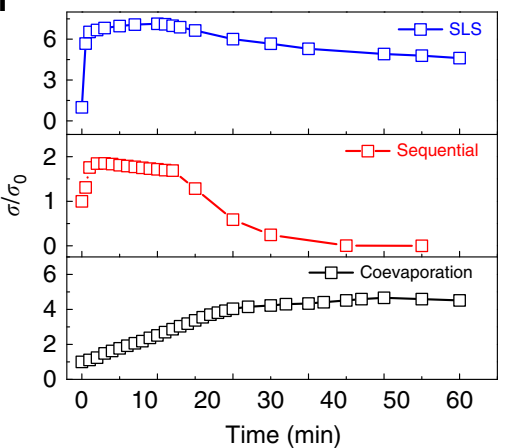

Fig. 1 Morphology, crystal structure and electrical conductivity of $\mathbf{C s S n l}_{\mathbf{3}}$ films. a-c Schematics of film deposition of the co-evaporation, sequential and SLS methods, respectively (before any annealing steps). d-f Corresponding scanning electron microscopy (SEM) images of the films after any annealing steps. $\mathbf{g}$ X-ray diffraction spectra of three types of thin film with lattice plane indices of the most prominent peaks in each case. Electrical conductivity of three types of perovskite film in nitrogen atmosphere (h) and in air (i).

structure. Co-evaporated films were mirror-black, characteristic of the $\mathrm{CsSnI}_{3}$ perovskite, whilst sequentially deposited and SLS films were red-brown, but became mirror-black after baking at $170{ }^{\circ} \mathrm{C}$ in nitrogen atmosphere (Supplementary Fig. 1). Scanning electron microscopy (SEM) revealed the dense polycrystalline morphology of the vapour-deposited $\mathrm{CsSnI}_{3}$ thin films (Fig. 1d-f). Sequential deposition produced perovskite thin films with around $1 \mu \mathrm{m}$ diameter grains, which were larger than grains in the coevaporated perovskite thin film (300-500 nm diameter). The SLS perovskite thin films also contained sub-micron grains, yet with a rougher surface morphology. As shown in Fig. 1g, X-ray diffraction patterns of $\mathrm{CsSnI}_{3}$ films made by all three deposition procedures showed features of the orthorhombic black phase, $\mathrm{B}-\gamma^{32}$ of $\mathrm{CsSnI}_{3}$, with peaks at $25.02^{\circ}$ and $29.15^{\circ}(2 \theta)$ corresponding to (220) and (202) planes, respectively. The sequentially processed films have a dominant peak at $29.15^{\circ}$, showing a preferred orientation of the (202) crystal plane parallel to the substrate. On the other hand, the co-evaporated films present preferential orientation of the (220) plane parallel to the surface. In the case of the SLS processed films, multiple peaks were observed, including both (220) and (202), indicating mixed orientations of crystallites in the film. The films are present in the B- $\gamma$ phase regardless of deposition method, which is confirmed with grazing-incidence X-ray diffraction (GIXRD) experiments (Supplementary Fig. 2), and there was no evidence of diffraction peaks associated with $\mathrm{Cs}_{2} \mathrm{SnI}_{6}$ or the precursor materials. The thickness of all films studied was between 250 and $300 \mathrm{~nm}$.
Electrical conductivity and stability. To characterise the electrical stability of our films, we performed time-dependent electrical conductivity measurements both in inert atmosphere $\left(\mathrm{N}_{2}\right.$ glovebox) and in air. $\mathrm{CsSnI}_{3}$ thin films from all three deposition methods showed high stability when tested in a $\mathrm{N}_{2}$ atmosphere, in fact showing a modest increase in electrical conductivity over a period of $1 \mathrm{~h}$ (Fig. 1h). In total over that period a reproducible increase in conductivity by a factor of 3.6 was observed for coevaporated films, 1.3 for sequentially evaporated and 1.2 for SLS films, reaching maximum conductivities of $8.5 \times 10^{-3}, 7.3$ and $6.8 \mathrm{~S} \mathrm{~cm}^{-1}$, respectively. When the thin films were exposed to air, $\sigma$ increased by a factor of 2 to 7 in all cases (Fig. 1i), which would be expected from a self-doping process during oxidation of $\mathrm{Sn}^{2+}$ to $\mathrm{Sn}^{4+33,34} . \sigma$ of co-evaporated thin films continuously increased for $45 \mathrm{~min}$, while $\sigma$ of sequentially deposited films increased for just $5 \mathrm{~min}$ before degradation caused a rapid decrease. The thin films deposited by the SLS method can sustain increases in $\sigma$ for $11 \mathrm{~min}$, reaching a value 7 times the initial one and remain reasonably stable afterwards, showing only $30 \%$ reduction in $\sigma_{\max }$ over the following $50 \mathrm{~min}$. $\mathrm{CsSnI}_{3}$ thin films deposited by SLS show a similar maximum electrical conductivity $\left(37.1 \mathrm{~S} \mathrm{~cm}^{-1}\right)$ to sequentially deposited films $\left(32.2 \mathrm{~S} \mathrm{~cm}^{-1}\right)$, which is $\sim 25$ times higher than the maximum for co-evaporated films $\left(1.2 \mathrm{~S} \mathrm{~cm}^{-1}\right)$, a value we consider too low for thermoelectric applications. Coevaporated films with dominate orientation (220) therefore show the best air stability but lowest electrical conductivity. Sequentially deposited films with dominate orientation (202) show a 

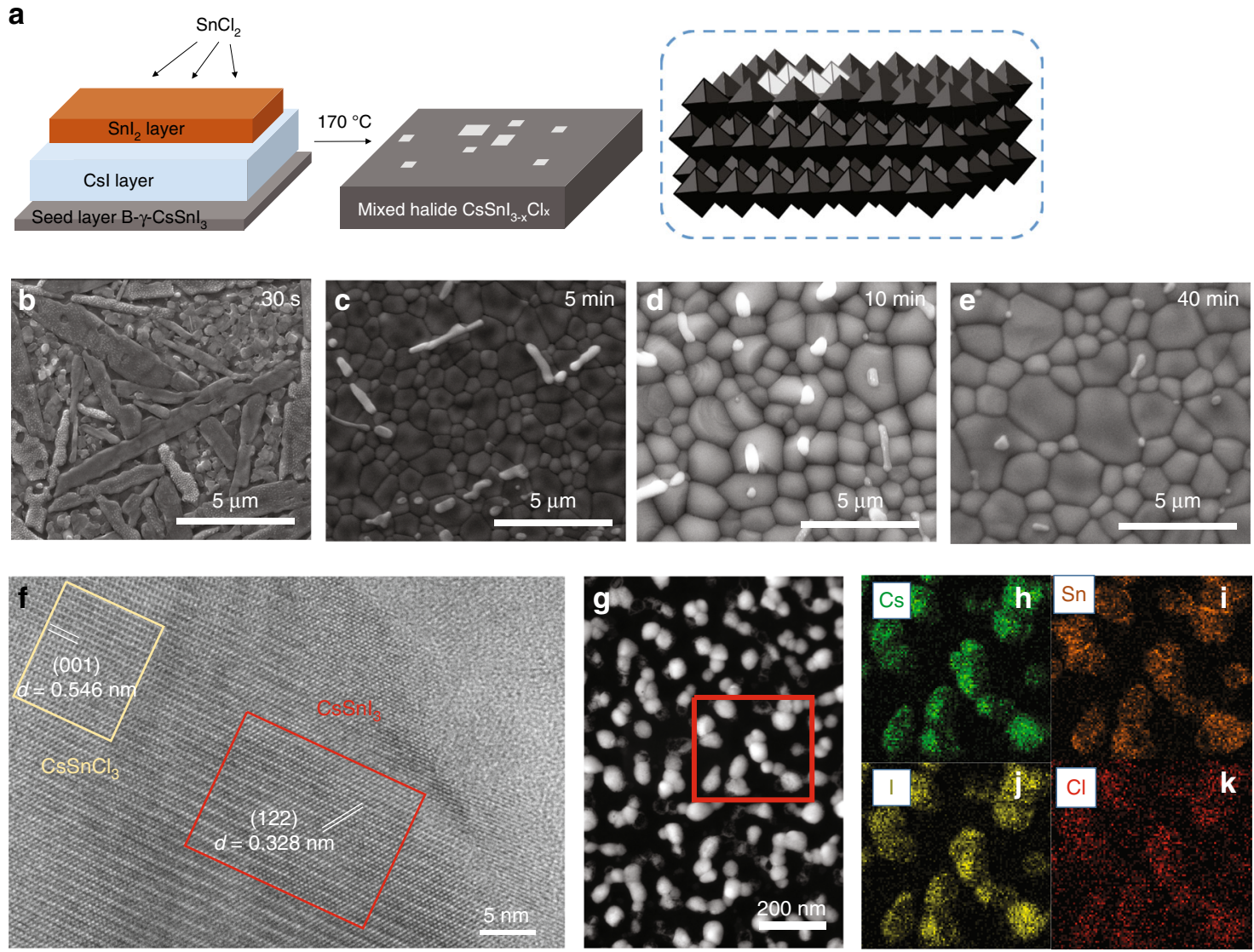

Fig. 2 Mixed halide $\mathbf{C s S n I}_{3-\mathbf{x}} \mathbf{C l}_{\mathbf{x}}$ perovskite morphology, structure and elemental distribution. a Schematic of the mixed halide perovskite deposition method. The white squares represent the $\mathrm{CsSnCl}_{3}$ structures in the top layers of the $\mathrm{B}-\boldsymbol{\gamma}-\mathrm{CsSn}_{3}$ perovskite films. $\mathbf{b}$-e SEM images of the morphological development of mixed halide perovskite films as a function of baking time. $\mathbf{f}$ High-resolution transmission electron microscopy (HRTEM) of mixed halide perovskite structures. The yellow square and red square correspond to $\mathrm{CsSnCl}_{3}$ and $\mathrm{CsSnl}{ }_{3}$ crystal lattices, respectively. $\mathbf{g} \mathrm{STEM}$-HAADF image of isolated grains of mixed halide $\mathrm{CsSnl}_{3-x} \mathrm{Cl}_{x}$ formed on an amorphous carbon support and (h-k) STEM-EDS elemental mapping in the area denoted by the red square in $(\mathbf{g})$.

poor air stability despite their larger grain sizes, but do have higher electrical conductivity. Previous work has shown that grain orientation can have a significant effect on degradation rates of halide perovskite films $\mathrm{s}^{35}$ and this is likely to be the case here. Since SLS films have enhanced stability compared with the sequentially deposited films, we chose SLS produced films as the platform from which to optimise the thermoelectric properties of $\mathrm{CsSnI}_{3}$ perovskites.

To further improve the air stability of SLS perovskite thin films, chloride was introduced in the deposition process, as mixed halide perovskites are known to exhibit improved air stability over analogous single-halide materials ${ }^{31,36}$. This was done by thermal deposition of a thin layer $(<20 \mathrm{~nm})$ of tin chloride $\left(\mathrm{SnCl}_{2}\right)$ on top of the $250-300-\mathrm{nm}$ thick SLS films prior to thermal annealing (schematic in Fig. 2a). Deposition was followed by baking under nitrogen atmosphere at $170^{\circ} \mathrm{C}$. An initial SEM investigation revealed an elongated grain structure on the top surface of our films (Fig. 2b) which was attribute to a pure $\mathrm{SnCl}_{2}$ phase. As baking progresses (Fig. 2c), the typical polycrystalline perovskite morphology with polygonal grains emerges, although a small number of the elongated crystals remain on top. On further baking, the remaining elongated crystals show reduced aspect ratio and the underlying perovskite grains merge into micronsized features (Fig. 2d), until after $40 \mathrm{~min}$ of baking (Fig. 2e), there was little evidence of the elongated crystals at all. The mixed halide films have XRD features similar to $\mathrm{CsSnI}_{3}$ with an absence of peaks that could be assigned to $\mathrm{SnCl}_{2}$ or $\mathrm{CsSnCl}_{3}$
(Supplementary Figs. 3 and 4). It should be noted that $\mathrm{SnCl}_{2}$ can sublime at $170^{\circ} \mathrm{C}$, so we used SEM and STEM combined with energy-dispersive X-ray spectroscopy (EDS) (Supplementary Fig. 5 and Fig. $2 \mathrm{f}-\mathrm{k}$, respectively) to confirm residual $\mathrm{Cl}$ incorporation into our samples. Furthermore, high-resolution transmission electron microscopy (HRTEM) of a single grain of our mixed halide perovskite (Fig. 2f), showed two regions of different crystal lattices (marked with red and yellow squares). The lattice spacing of $0.328 \mathrm{~nm}$ measured in the red region corresponds to the $\mathrm{CsSnI}_{3}$ crystal (122) plane, whilst the lattice spacing of $0.546 \mathrm{~nm}$ measured in the yellow region corresponds to the (001) plane of the $\mathrm{CsSnCl}_{3}$ cubic lattice. This indicates a degree of nanoscale phase separation between chlorine-rich and iodine rich phases within perovskite grains. The absence of $\mathrm{CsSnCl}_{3}$ features in the XRD spectra is due to the low concentration of $\mathrm{Cl}$ in our films. Corroborating evidence for the incorporation of chlorine into perovskite structures is provided by X-ray photoelectron spectroscopy (XPS), with the $\mathrm{Cl} 2 p$ peak of our mixed halide films showing a significant broadening compared with $\mathrm{SnCl}_{2}$ (Supplementary Fig. 6) ${ }^{31}$. Moreover, we used XPS to get a depth profile of the Cl concentration in our films (Supplementary Fig. 7), finding that $\mathrm{Cl}$ was present in the top layer, penetrating only a few nanometres into the bulk. We could not detect any chlorine by XPS at depths larger than $10 \mathrm{~nm}$ from the film surface. In what follows, we studied 0.5, 1, 3 and 5\% $\mathrm{SnCl}_{2}$ mixed halide $\mathrm{CsSnI}_{3-\mathrm{x}} \mathrm{Cl}_{\mathrm{x}}$ perovskite films. The percentage we use refers to the mass of $\mathrm{SnCl}_{2}$ relative to $\mathrm{SnI}_{2}$ in our thin films 
before the baking step. The final atomic $\%$ of $\mathrm{Cl}$ in the film will be much lower.

To demonstrate the improved stability of our mixed halide perovskite films, we studied the quenching of the optical absorption peak at $420 \mathrm{~nm}$ (Supplementary Fig. 8). 5\% Cldoped SLS films show enhanced stability, with just $3 \%$ quenching of the $420 \mathrm{~nm}$ peak after $100 \mathrm{~min}$ air exposure, whereas SLS $\mathrm{CsSnI}_{3}$ films without Cl-doping showed a $40 \%$ quenching of the peak under the same conditions. In fact, in terms of their optical properties, the $5 \% \mathrm{Cl}$-doped SLS films are more stable than undoped co-evaporated films, showing less than half of the quenching of the absorption after $500 \mathrm{~min}$ in air.

Quantitative analysis of Sn oxidation states. As the origin of high conductivity in tin halide perovskites comes from hole doping due to the oxidation of $\mathrm{Sn}^{2+}$ to $\mathrm{Sn}^{4+}$, we used XPS analysis to probe the oxidation state of $\mathrm{Sn}$ in our films. Shifts in the Sn $3 d_{5 / 2}$ peak are relatively modest as a function of oxidation state (Supplementary Fig. 9), so we focussed on the Auger region of the spectrum. Since Auger electron spectroscopy (AES) probes three-electron process, it is a much more sensitive measure of oxidation state. We did this as a function of depth in $\mathrm{CsSnI}_{3-\mathrm{x}} \mathrm{Cl}_{\mathrm{x}}$ films $\left(1 \% \mathrm{SnCl}_{2}\right)$ which had undergone a short air exposure (Fig. 3a). The Sn MNN AES spectrum shows a broad line shape, including several Sn MNN peaks (fitting curves labelled $a, b, c$ and $d$, with details in Supplementary Table 1). In the $\mathrm{Sn}^{0}$ metal $\mathrm{M}_{5} \mathrm{~N}_{4,5} \mathrm{~N}_{4,5}$ AES spectrum reported by Barlow et al. ${ }^{37},{ }^{1} \mathrm{~S}_{0}$ has a peak at a kinetic energy of $421.2 \mathrm{eV}$, and showed a large broadening after oxidation. In our case, the ${ }^{1} S_{0}$ peak (fitted curve $a$ ) is broad, confirming the absence of $\mathrm{Sn}^{0}$ states. Fitted curve $b$ (425-430 eV) includes multiplet splitting of the ${ }^{1} \mathrm{G}_{4},{ }^{3} \mathrm{P}_{2},{ }^{3} \mathrm{~F}_{2,3}$ and ${ }^{3} \mathrm{~F}_{4}$ states (Supplementary Table 1). This broad peak shifts to higher kinetic energy with increasing etching depth, corresponding to oxidation in the top layer compared with the bulk ${ }^{38-40}$. This is even more evident from peak $c$, which is linked to $\mathrm{Sn}^{4+}$ states $^{38-40}$ and is prominent at the surface, but disappears completely within an etching depth of $7.5 \mathrm{~nm}$ (Fig. 3b).

The modified Auger parameter $\left(\alpha^{\prime}\right)$ can be used for a more robust identification of chemical states of elements in molecules or solids, and is not susceptible to shifts caused by sample charging ${ }^{38-41}$. It is defined as the sum of the kinetic energy of a core-core-core Auger line, $E_{\mathrm{k}}$, and the binding energy, $E_{\mathrm{b}}$, of a core electron, $\alpha^{\prime}=E_{\mathrm{k}}+E_{\mathrm{b}}$ and can be viewed more intuitively if plotted in a Wagner format ${ }^{42}$ (a plot of $E_{\mathrm{k}}$ versus $E_{\mathrm{b}}$ recorded from all chemical states of the atom). The Wagner plot in Fig. 3c, combines our own data (as a function of depth) with some literature references for $\mathrm{Sn}^{0}, \mathrm{Sn}^{2+}$ and $\mathrm{Sn}^{4+}$ states ${ }^{39,40,43}$, and clearly illustrates a mixture of $\mathrm{Sn}^{2+}$ and $\mathrm{Sn}^{4+}$ oxidation states in our film (Sn core binding energy, Auger kinetic energy and Auger parameters detailed in Supplementary Tables 2-3 and Supplementary Fig. 10 $)^{44}$. Moreover, there is a progressive change from majority $\mathrm{Sn}^{4+}$ states at the surface of the film to $\mathrm{Sn}^{2+}$ at a depth of $10 \mathrm{~nm}$, further evidence that the oxidation process only occurs in the top $7.5 \mathrm{~nm}$ of the film, the same region of the film that incorporates chlorine dopants.

From this, we can conclude that the top surface layer of the mixed $\mathrm{CsSnI}_{3-\mathrm{x}} \mathrm{Cl}_{\mathrm{x}}$ acts as a sacrificial layer where initial oxidation occurs. This layer provides hole doping to the bulk (vide infra) from the surface $\mathrm{Sn}^{4+}$ species. This mechanism, which separates the doping layer from the transport region, minimises the structural impact of doping on charge mobility, and enables our mixed halide perovskite structure to present high electrical conductivity whilst retaining a reasonable degree of air stability.

Thermoelectric properties of $\mathrm{CsSnI}_{3-x} \mathrm{Cl}_{x}$ thin films. We performed thermoelectric property measurements as shown in Fig. 4a-f. The temperature dependence of $\sigma$ and the sign of $\alpha$ for $1 \% \mathrm{Cl}$-doped $\mathrm{CsSnI}_{3-\mathrm{x}} \mathrm{Cl}_{\mathrm{x}}$ in the range $290-360 \mathrm{~K}$ (Fig. $4 \mathrm{a}, \mathrm{b}$ ) indicates band-like transport and that the majority charge carriers are holes, as reported previously for $\mathrm{CsSnI}_{3}$ single crystal nanowires $^{22}$, validating the high quality of our mixed halide perovskite films. $\alpha$ increases approximately linearly with temperature due to the shift of the Fermi level away from the valence band, following the Fermi-Dirac distribution within the mobility edge model of the Seebeck coefficient for a heavily doped semiconductors ${ }^{45}$.

We can fine tune the electrical conductivity of our films by exposing them to air ( $3 \mathrm{~min}$ at a time) to further oxidise $\mathrm{Sn}^{2+}$ to $\mathrm{Sn}^{4+}$. At room temperature, initial electrical conductivity, $\sigma_{0}$, was

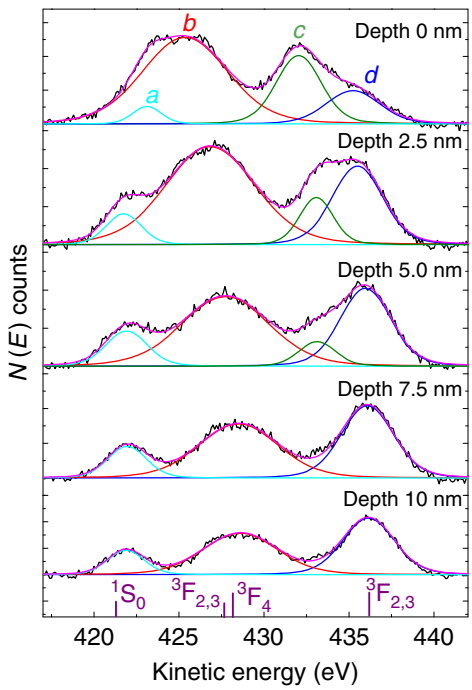

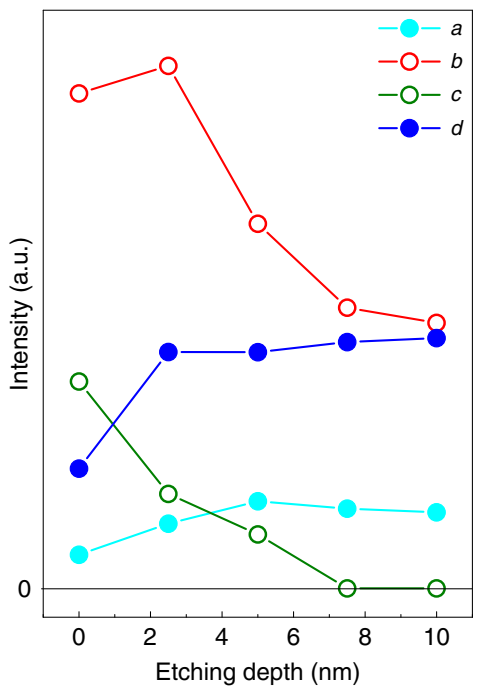

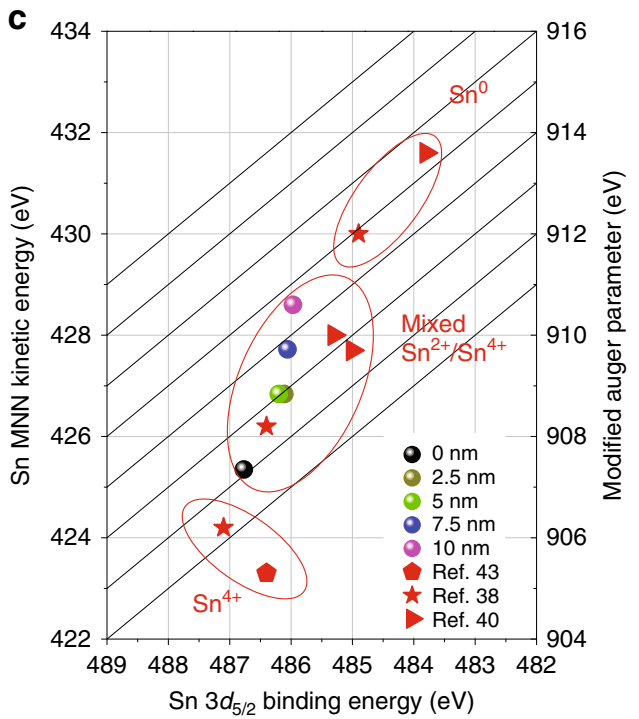

Fig. $3 \mathbf{S n}$ oxidation state in $\mathbf{1} \% \mathbf{C l}_{\text {-doped }} \mathbf{C s S n l}_{\mathbf{3}-\mathbf{x}} \mathbf{C l}_{\mathbf{x}}$ perovskite thin films. a Auger electron spectra of $\mathrm{Sn}$ MNN at different etching depths from 0 to $10 \mathrm{~nm}$. b Photoelectron counts of fitted curves in (a) as a function of etching depth. c Sn $3 d_{5 / 2}$ Wagner plot with the modified Auger parameter of our samples (circles) and reference values for $\mathrm{Sn}^{0}, \mathrm{Sn}^{2+}$ and $\mathrm{Sn}^{4+}$. 

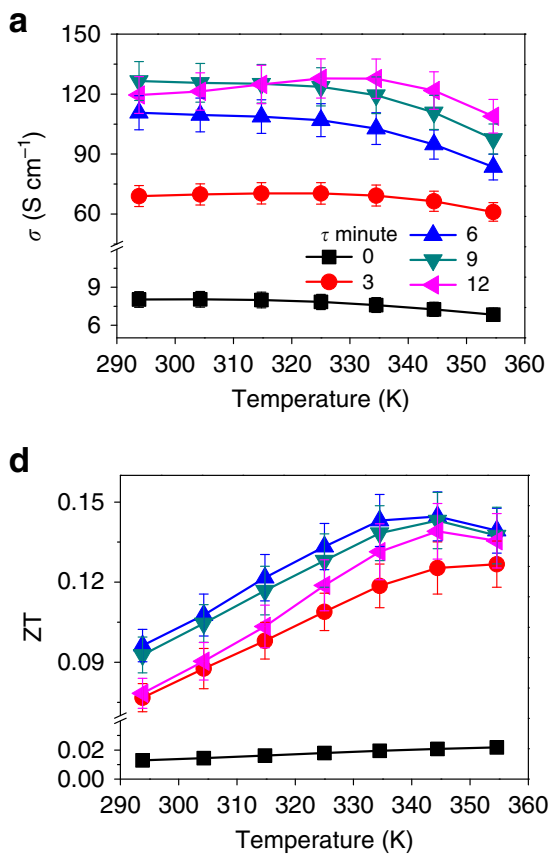
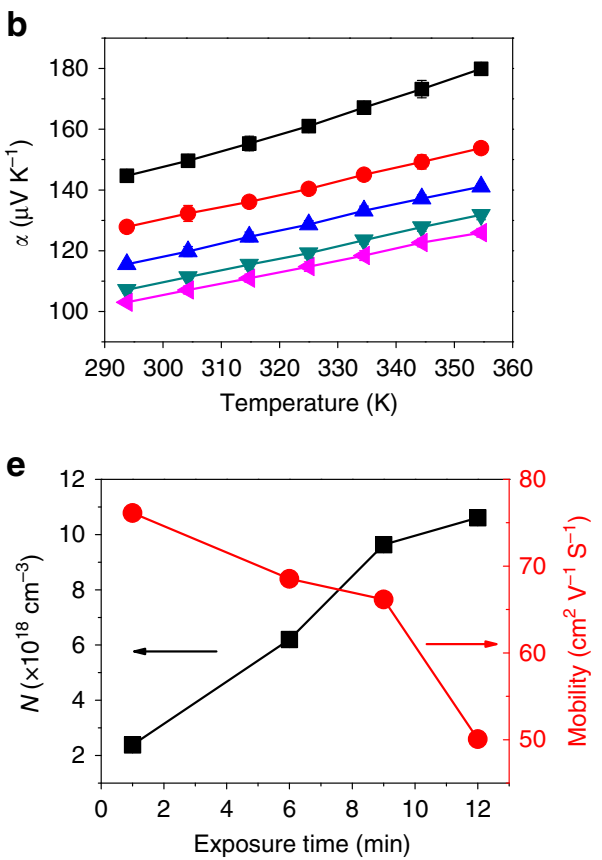
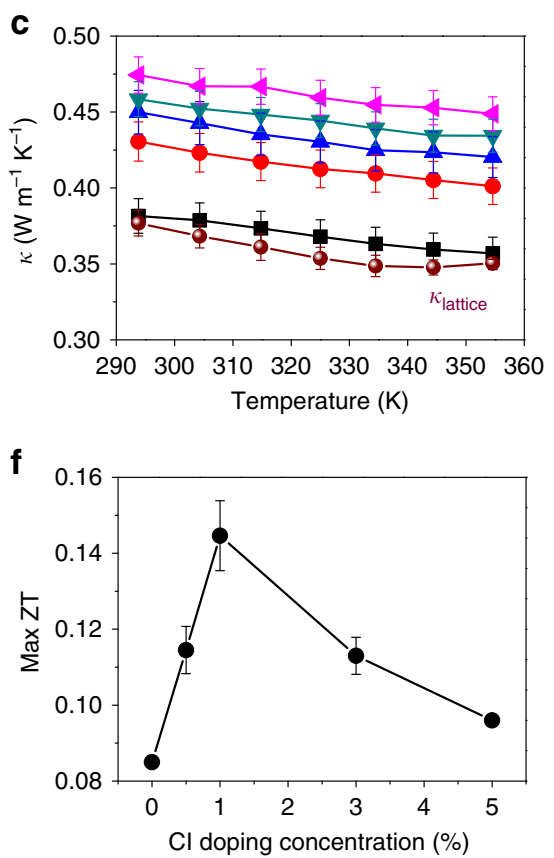

Fig. 4 Thermoelectric properties of $\mathbf{1} \%$ mixed $\mathbf{C s S n I}_{\mathbf{3}-\mathbf{x}} \mathbf{C l}_{\mathbf{x}}$ perovskite thin films. Temperature dependence of electrical conductivity $\sigma$ (a), Seebeck coefficient $\alpha(\mathbf{b})$, thermal conductivity $\kappa_{\text {total }}(\mathbf{c})$ and figure-of-merit, ZT $(\mathbf{d})$. The differently coloured curves represent different degrees oxidation according to the legend in plot (a). Error bars in (b) are too small to be visible, typically representing $<2 \%$ of the value. e Charge carrier density and Hall mobility as a function of air exposure time. $\mathbf{f}$ Maximum figure-of-merit, $\mathrm{ZT}$, as a function of $\mathrm{SnCl}_{2}$ incorporation.

$8.0 \pm 0.6 \mathrm{~S} \mathrm{~cm}^{-1}$ (Fig. $4 \mathrm{a}$ ) and it dramatically increased to $69.0 \pm$ $5.2 \mathrm{~S} \mathrm{~cm}^{-1}$ after a further air exposure $\left(\sigma_{3}\right)$, and saturated at $126.5 \pm 9.7 \mathrm{~S} \mathrm{~cm}^{-1}$ after $9 \mathrm{~min}$ air exposure $\left(\sigma_{9}\right)$. Further air exposure lead to a slower, but steady decrease in electrical conductivity $\left(\sigma_{12}=119.6 \pm 9.2 \mathrm{~S} \mathrm{~cm}^{-1}\right)$. The dependence of $\sigma$ on air exposure time comes from the competition between the enhanced charge carrier concentration owing to self-doping from $\mathrm{Sn}^{4+}$ species and reduced carrier mobility owing to defects caused by air exposure, which can take the form of degradation in the bulk or at the grain boundaries during the oxidation process, or even increased ionised impurity scattering.

The Seebeck coefficient, $\alpha$, in Fig. $4 \mathrm{~b}$ shows a steady decrease with exposure time (at room temperature, $\alpha_{0}=144.7 \pm 1.5 \mu \mathrm{V} \mathrm{K}^{-1}$, and after $12 \mathrm{~min}$ air exposure $\alpha_{12}=103.0 \pm 1.0 \mu \mathrm{V} \mathrm{K}^{-1}$ ), consistent with a steady increase in the charge concentration shifting the Fermi energy level, $E_{\mathrm{f}}$, towards the valence band. The observation that the Seebeck coefficient continues to decrease with air exposure when the electrical conductivity has already peaked is further evidence that the degradation in conductivity after extended exposure to air is due to mobility lowering processes. To verify this hypothesis, we used Hall measurements to determine the charge carrier concentration as a function of air exposure, showing an increase with air exposure from $2.38 \times 10^{18}$ to $1.06 \times 10^{19} \mathrm{~cm}^{-3}$ after 12 min (Fig. 4e). Meanwhile, the Hall mobility decreases from an initial value of 76.1 to $50.1 \mathrm{~cm}^{2} \mathrm{~V}^{-1} \mathrm{~s}^{-1}$ after oxidation. We note that in Lee et al.'s work ${ }^{22}$, lower $\alpha\left(79 \mu \mathrm{V} \mathrm{K}^{-1}\right)$ at room temperature with higher $\sigma\left(282 \mathrm{~S} \mathrm{~cm}^{-1}\right)$ indicates a higher level of self-doping, whereas our control of oxidation level allows us to precisely tune the $\alpha / \sigma$ ratio and ultimately optimise ZT.

The measured temperature-dependent thermal conductivity for $1 \% \mathrm{SnCl}_{2}$ perovskite thin films is presented in Fig. $4 \mathrm{c}$. At room temperature after a minimal air exposure of $30 \mathrm{~s}$, the thermal conductivity is $0.38 \pm 0.01 \mathrm{~W} \mathrm{~m}^{-1} \mathrm{~K}^{-1}$ and it increases with air exposure to $0.47 \pm 0.01 \mathrm{~W} \mathrm{~m}^{-1} \mathrm{~K}^{-1}$. To obtain the lattice thermal conductivity $\kappa_{\text {lattice }}$ from the measured $\kappa_{\text {total }}\left(=\kappa_{\text {lattice }}+\right.$ $\kappa_{\text {electronic }}$ ), we plotted $\kappa_{\text {total }}$ as a function of $\sigma$ (Supplementary
Fig. 11). The electronic thermal conductivity, $\kappa_{\text {electronic }}$ is described by Wiedemann-Franz law $\left(\kappa_{\text {electronic }}=\sigma L T\right)$, enabling us to determine $\kappa_{\text {lattice }}$ and the Lorentz number, $L$, from the intercept and slope, respectively, of a linear fit. We note that since electrical doping is provided by a thin $(<10 \mathrm{~nm})$ surface layer, we can assume that the lattice thermal conductivity in the bulk of the film is not strongly affected by the doping process, which is a requirement for this analysis. We found $\kappa_{\text {lattice }}=$ $0.38 \pm 0.01 \mathrm{~W} \mathrm{~m}^{-1} \mathrm{~K}^{-1}$ at room temperature, which is consistent with Lee's work $\left(0.38 \pm 0.04 \mathrm{~W} \mathrm{~m}^{-1} \mathrm{~K}^{-1}\right)$, and extract a Lorentz number of $(2.40 \pm 0.33) \times 10^{-8} \mathrm{~W} \Omega \mathrm{K}^{-2}$ at room temperature or an average of $(2.26 \pm 0.13) \times 10^{-8} \mathrm{~W} \Omega \mathrm{K}^{-2}$ over the full temperature range, close to the Sommerfeld value for free electrons. Furthermore, we can confirm that polycrystalline $\mathrm{CsSnI}_{3-\mathrm{x}} \mathrm{Cl}_{\mathrm{x}}$ thin films exhibit a temperature dependence of $\kappa_{\text {lattice }}$ that is consistent with the Calloway model ${ }^{46}$, with Umklapp scattering processes dominating in this temperature range, as has been reported for methylammonium lead iodide perovskites $^{21,47}$.

Finally, the thermoelectric figure-of-merit, ZT, of our $\mathrm{CsSnI}_{3-\mathrm{x}} \mathrm{Cl}_{\mathrm{x}}$ perovskite films increases for oxidation time, $\tau$, in the range $0-6 \mathrm{~min}$, and then decreases for $\tau$ more than 6 min, as shown in Fig. $4 \mathrm{~d}$. The largest ZT is $0.14 \pm 0.01$ at $345 \mathrm{~K}$ for $1 \% \mathrm{CsSnI}_{3-\mathrm{x}} \mathrm{Cl}_{\mathrm{x}}$, a factor of 7 higher compared with that of the $\tau=0$ sample $(\mathrm{ZT}=0.02$ at $355 \mathrm{~K})$. The figure-of-merit shows a $32 \%$ reduction after $10 \mathrm{~h}$ in air, and a $30 \%$ reduction after 10 days storage in nitrogen atmosphere (Supplementary Figs. 12 and 13). We also note that thinner films showed higher electrical conductivities, but no improvement in ZT (Supplementary Fig. 14). This high degree of control over ZT through tuning of $\sigma$ and $\alpha$ indicates the effectiveness of self-doping in the thermoelectric performance of Sn-halide perovskites. Interestingly, the maximum $\mathrm{ZT}$ is a function of the degree of Cl-doping (Fig. 4f), with a sharp increase of $\mathrm{ZT}_{\max }$ from 0.07 at $0 \% \mathrm{Cl}$ to a peak of $\mathrm{ZT}_{\max }=0.14 \pm 0.01$ at $1 \% \mathrm{Cl}$ and steady decrease upon further Cl-inclusion. In parallel, we observe that 
the Seebeck coefficient decreases as a function of Cl-doping (Supplementary Figs. 15, 18), implying that the more heavily $\mathrm{Cl}$-doped the films are, the higher the charge carrier density that is achieved. This is further evidence that the chlorine-rich surface layer is acting not only as a protective layer slowing down oxidation of the underlying $\mathrm{CsSnI}_{3}$, but also as a sacrificial source of holes in this system that are donated from the surface to the bulk. This separation of the dopants from the charge transport channel prevents the introduction of scattering defects in the transport channel which can reduce charge mobility, and is the reason that our $\mathrm{Cl}$-doped films can achieve up to four times the maximum electrical conductivity of our pristine $\mathrm{CsSnI}_{3}$ films (Fig. 1 and Supplementary Fig. 18).

\section{Discussion}

Our work sheds light on optimisation strategies of halide perovskites for thermoelectrics, with wider implications for the development of halide perovskite films with targeted properties across other application areas such as photovoltaics, photodetectors, thin film transistors and light-emitting diodes. We have demonstrated a number of thermal vapour deposition methods for the formation of high quality $\mathrm{CsSnI}_{3}$ thin films from its precursor materials. These films are self-doping through oxidation of $\mathrm{Sn}^{2+}$ to $\mathrm{Sn}^{4+}$, and we have shown that the stability and electrical conductivity of the films is highly dependent on whether a sequential or co-evaporation process is adopted, with the former offering higher electrical conductivity and the latter higher stability. For this reason, we developed a hybrid of the two approaches (SLS) to offer a suitable platform from which to optimise thermoelectric properties. Beyond this and building on knowledge that mixed halide approaches can improve atmospheric stability of halide perovskites, we adopted a unique approach to chlorine doping of our $\mathrm{CsSnI}_{3}$ films, that results in substitution of chlorine into a perovskite crystal lattice in a region $<10 \mathrm{~nm}$ from the surface. We have shown that the $\mathrm{Cl}$-dopants not only enhance stability but simultaneously act as a sacrificial source of free charges. The electrical doping is therefore coming from the outer atomic layers of the film, but dopes to the pristine bulk, thus dividing the film into a thin doping layer and a thicker charge transport channel and ensuring that the introduction of dopants does not degrade mobility in the transport channel.

The accessible free charge carrier concentration is therefore determined by the $\mathrm{Cl}$ concentration and by tuning this in combination with the degree of oxidation, we have optimised thermoelectric performance, achieving $\mathrm{ZT}=0.14 \pm 0.01$, and verified that the Wiedemann-Franz law is valid in these materials with a Lorenz number similar to the Sommerfeld value for free electrons. These results are important in identifying routes to develop the halide perovskite class of materials for thermoelectric applications, but the process of their optimisation for thermoelectrics has revealed a deeper understanding of their thermal and electrical transport properties, as well as strategies for controlled doping, which has implications across all areas of their application. The potential advantages of halide perovskite materials for thermoelectrics are elemental abundance as well as mechanical flexibility, solution processability and large area scalability. Finally, we note that the stability of this tin based perovskite material could be further improved by adding a strong reductant with favourable Goldschmidt tolerance into the structure, pursuing layered structures, surface passivation or adopting mixed metal approaches.

\section{Methods}

Film deposition. We present three type of evaporated films: co-evaporated, sequentially evaporated and seed layer plus sequential deposition (SLS). For coevaporated films, tin (II) iodide $\left(\mathrm{SnI}_{2}, 99.99 \%\right.$, Sigma-Aldrich) and caesium iodide
(CsI, 99.99\%, Sigma-Aldrich) were simultaneously deposited at $10^{-7}$ mbar. The deposition rate was $1 \AA^{-1}$ for $\mathrm{SnI}_{2}$ (achieved with a crucible temperature of $160^{\circ} \mathrm{C}$ ) and $3 \AA \mathrm{s}^{-1}$ for CsI (achieved with a crucible temperature of $430^{\circ} \mathrm{C}$ ). The mirror-black films were directly obtained from the co-evaporation methods without annealing. For the sequential deposition methods, $\mathrm{SnI}_{2}$ was thermally evaporated at $10^{-7} \mathrm{mbar}$ at $2 \AA \mathrm{s}^{-1}\left(170^{\circ} \mathrm{C}\right)$, followed by CsI at $6 \AA \mathrm{s}^{-1}\left(450^{\circ} \mathrm{C}\right)$. The initially redbrown thin films were removed from the vacuum chamber for baking at $170{ }^{\circ} \mathrm{C}$ in nitrogen atmosphere. Upon baking, the appearance of the films became mirrorblack indicating that $\mathrm{CsSnI}_{3}$ thin films were successfully fabricated. For SLS films, a $50 \mathrm{~nm}$ co-evaporated layer was first deposited as a seed layer. Above the seed layer followed a layer deposited by the sequential method without breaking vacuum. Dark brown films were obtained from the SLS method, forming mirror-black $\mathrm{CsSnI}_{3}$ films upon baking at $170{ }^{\circ} \mathrm{C}$. For mixed halide perovskite samples, tin (II) chloride $\left(\mathrm{SnCl}_{2}\right.$, 99.99\%, Sigma-Aldrich) was evaporated at $0.5 \AA \mathrm{s}^{-1}$ (achieved with a crucible temperature of $130^{\circ} \mathrm{C}$ ) on top of SLS films (which had not been baked) without breaking vacuum. The mixed halide films were also baked at $170{ }^{\circ} \mathrm{C}$ in nitrogen atmosphere to form mirror-black mixed halide perovskite films.

Scanning electron microscopy. The surface morphology of the films was performed on a field-emission scanning electron microscope (FEI Inspect-F).

Optical absorption. UV-Vis absorption spectra were measured with Shimadzu UV-2600 spectrophotometer, using $10 \mathrm{~min}$ intervals for time-dependent air stability studies.

X-ray diffraction. X-ray diffraction was performed on a Siemens D5000 X-Ray Powder diffractometer using a Cu Ka source $(\lambda=1.54 \AA)$.

Grazing-incidence $\mathbf{X}$-ray diffraction. GIXRD measurements were performed at the XRD1 beamline of the ELETTRA synchrotron facility in Trieste (Italy). The X-ray beam had a wavelength of $0.7 \AA$ and a beam size of $200 \times 200 \mu \mathrm{m}^{2}$. 2D-GIWAXS images were collected by using $2 \mathrm{M}$ Pilatus silicon pixel X-ray detector (DECTRIS Ltd.) positioned perpendicular to the incident beam, at a distance of $260 \mathrm{~mm}$ from the sample. The grazing incident angle was fixed at $\alpha_{\mathrm{i}}=$ $0.5^{\circ}$ to probe the full thickness of the film.

Scanning transmission electron microscopy-energy-dispersive $X$-ray spectroscopy. Transmission electron microscopy (TEM) and high-resolution TEM imaging was carried out on a Tecnai G ${ }^{2}$ F20 S-TWIN at $200 \mathrm{kV}$. High angle annular dark field scanning transmission electron microscopy (HAADF-STEM) imaging and energy-dispersive X-ray spectroscopy (EDS) elemental mapping were performed on a JEM-ARM $200 \mathrm{~F}$ at $200 \mathrm{kV}$. The TEM specimen fabrication was by the evaporation process of $\mathrm{CsSnI}_{3-\mathrm{x}} \mathrm{Cl}_{\mathrm{x}}$ perovskite mentioned in the Film deposition section onto a copper grid with an amorphous carbon film on top.

X-ray photoelectron spectroscopy. XPS was performed on Thermo Scientific K-Alpha X-ray photoelectron spectrometer with a monochromatic Al Ka X-ray source under high vacuum $\left(2 \times 10^{-8} \mathrm{mbar}\right)$. Etching of the films for depth profiling was by in situ sputtering at room temperature using a beam of $3 \mathrm{keV} \mathrm{Ar}^{+}$ions. The etching depth profile was calculated from the etching time required to etch through to the silicon substrate. Fitting was performed on the CasaXPS package, incorporating Voigt line shapes and a Shirley background.

Thermoelectric properties measurement. In-plane thermoelectric properties $(\sigma, \kappa$ and $\alpha$ ) were measured simultaneously on the same sample with a Linseis Thin Film Analyser (described elsewhere ${ }^{48-50}$ ). In this measurement geometry, in-plane thermal conductivity is measured on a suspended $\mathrm{SiN}$ membrane by a $3-\omega$ method. Electrical conductivity is measured by the van der Pauw method with four needle like electrodes at the four corners of the films. The Seebeck coefficient measurement uses a thermometer and a heater on the suspended membrane to achieve a temperature gradient (schematised in the Supplementary Fig. 19). Samples fabricated in the glovebox were transferred to the Linseis Thin Film Analyser with $<2 \mathrm{~min}$ exposure to air. The humidity in lab was around $40 \%$. All measurements were performed under vacuum and in the dark. Hall effect measurements were performed on PPMS-9 from Quantum Design Inc. When we wished to partially oxidise the films, the measurement chamber was refilled with air to atmospheric pressure for a designated time before pumping down again for the next measurement.

\section{Data availability}

The data that support the findings of this work are available from the corresponding author on request.

Received: 14 June 2019; Accepted: 26 November 2019; Published online: 17 December 2019 


\section{References}

1. Kojima, A., Teshima, K., Shirai, Y. \& Miyasaka, T. Organometal halide perovskites as visible-light sensitizers for photovoltaic cells. J. Am. Chem. Soc. 131, 6050-6051 (2009).

2. Burschka, J. et al. Sequential deposition as a route to high-performance perovskite-sensitized solar cells. Nature 499, 316-319 (2013).

3. Lee, M. M., Teuscher, J., Miyasaka, T., Murakami, T. N. \& Snaith, H. J. Efficient hybrid solar cells based on meso-superstructured organometal halide perovskites. Science 338, 643-647 (2012).

4. Best Research-Cell Efficiency Chart. https://www.nrel.gov/pv/cell-efficiency. html (2019).

5. Dong, Q. F. et al. Electron-hole diffusion lengths $>175 \mu \mathrm{m}$ in solution-grown $\mathrm{CH}_{3} \mathrm{NH}_{3} \mathrm{PbI}_{3}$ single crystals. Science 347, 967-970 (2015).

6. Shi, D. et al. Low trap-state density and long carrier diffusion in organolead trihalide perovskite single crystals. Science 347, 519-522 (2015).

7. Akkerman, Q. A. et al. Solution synthesis approach to colloidal cesium lead halide perovskite nanoplatelets with monolayer-level thickness control. J. Am. Chem. Soc. 138, 1010-1016 (2016).

8. Shamsi, J. et al. Colloidal synthesis of quantum confined single crystal $\mathrm{CsPbBr}_{3}$ nanosheets with lateral size control up to the micrometer range. J. Am. Chem. Soc. 138, 7240-7243 (2016).

9. Song, J. Z. et al. Monolayer and few-layer all-inorganic perovskites as a new family of two-dimensional semiconductors for printable optoelectronic devices. Adv. Mater. 28, 4861-4869 (2016).

10. Dou, L. T. et al. Atomically thin two-dimensional organic-inorganic hybrid perovskites. Science 349, 1518-1521 (2015).

11. Schmidt, L. C. et al. Nontemplate synthesis of $\mathrm{CH}_{3} \mathrm{NH}_{3} \mathrm{PbBr}_{3}$ perovskite nanoparticles. J. Am. Chem. Soc. 136, 850-853 (2014).

12. Zou, W. et al. Minimising efficiency roll-off in high-brightness perovskite light-emitting diodes. Nat. Commun. 9, 608 (2018).

13. Xing, J. et al. Color-stable highly luminescent sky-blue perovskite lightemitting diodes. Nat. Commun. 9, 3541 (2018).

14. Gong, X. et al. Electron-phonon interaction in efficient perovskite blue emitters. Nat. Mater. 17, 550-556 (2018)

15. Shrestha, S. et al. High-performance direct conversion X-ray detectors based on sintered hybrid lead triiodide perovskite wafers. Nat. Photon 11, 436-440 (2017).

16. Pan, W. C. et al. $\mathrm{Cs}_{2} \mathrm{AgBiBr}_{6}$ single-crystal X-ray detectors with a low detection limit. Nat. Photon 11, 726-732 (2017).

17. Zhu, H. et al. Lead halide perovskite nanowire lasers with low lasing thresholds and high quality factors. Nat. Mater. 14, 636-642 (2015).

18. Yakunin, S. et al. Low-threshold amplified spontaneous emission and lasing from colloidal nanocrystals of caesium lead halide perovskites. Nat. Commun. 6, 8056 (2015)

19. Hata, T., Giorgi, G. \& Yamashita, K. The effects of the organic-inorganic interactions on the thermal transport properties of $\mathrm{CH}_{3} \mathrm{NH}_{3} \mathrm{PbI}_{3}$. Nano Lett. 16, 2749-2753 (2016).

20. Yue, S. Y., Zhang, X. L., Qin, G. Z., Yang, J. Y. \& Hu, M. Insight into the collective vibrational modes driving ultralow thermal conductivity of perovskite solar cells. Phys. Rev. B 94, 115427 (2016).

21. Mettan, X. et al. Tuning of the thermoelectric figure of merit of $\mathrm{CH}_{3} \mathrm{NH}_{3} \mathrm{MI}_{3}$ $(\mathrm{M}=\mathrm{Pb}, \mathrm{Sn})$ photovoltaic perovskites. J. Phys. Chem. C. 119, 11506-11510 (2015).

22. Lee, W. et al. Ultralow thermal conductivity in all-inorganic halide perovskites. Proc. Natl Acad. Sci. USA 114, 8693-8697 (2017).

23. He, Y. P. \& Galli, G. Perovskites for solar thermoelectric applications: a first principle study of $\mathrm{CH}_{3} \mathrm{NH}_{3} \mathrm{Al}_{3}(\mathrm{~A}=\mathrm{Pb}$ and $\mathrm{Sn})$. Chem. Mater. 26, 5394-5400 (2014).

24. Walsh, A., Scanlon, D. O., Chen, S., Gong, X. G. \& Wei, S.-H. Self-regulation mechanism for charged point defects in hybrid halide perovskites. Angew. Chem. Int. Ed. 54, 1791-1794 (2015).

25. Brandt, R. E., Stevanović, V., Ginley, D. S. \& Buonassisi, T. Identifying defecttolerant semiconductors with high minority-carrier lifetimes: beyond hybrid lead halide perovskites. MRS Commun. 5, 265-275 (2015).

26. Abdelhady, A. L. et al. Heterovalent dopant incorporation for bandgap and type engineering of perovskite crystals. J. Phys. Chem. Lett. 7, 295-301 (2016).

27. Mitzi, D. B., Feild, C. A., Harrison, W. T. A. \& Guloy, A. M. Conducting tin halides with a layered organic-based perovskite structure. Nature 369, 467-469 (1994).

28. Takahashi, Y., Hasegawa, H., Takahashi, Y. \& Inabe, T. Hall mobility in tin iodide perovskite $\mathrm{CH}_{3} \mathrm{NH}_{3} \mathrm{SnI}_{3}$ : evidence for a doped semiconductor. J. Solid State Chem. 205, 39-43 (2013).

29. Saini, S., Baranwal, A., Yabuki, T., Hayase, S. \& Miyazaki, K. Growth of halide perovskites thin films for thermoelectric applications. MRS Adv. 4, 1719-1725 (2019).

30. Wang, P. Y. et al. Solvent-controlled growth of inorganic perovskite films in dry environment for efficient and stable solar cells. Nat. Commun. 9, 2225 (2018).
31. Marshall, K. P., Walker, M., Walton, R. I. \& Hatton, R. A. Enhanced stability and efficiency in hole-transport-layer-free $\mathrm{CsSnI}_{3}$ perovskite photovoltaics. Nat. Energy 1, 16178 (2016).

32. Chung, I. et al. $\mathrm{CsSnI}_{3}$ : semiconductor or metal? High electrical conductivity and strong near-infrared photoluminescence from a single material. High hole mobility and phase-transitions. J. Am. Chem. Soc. 134, 8579-8587 (2012).

33. Takahashi, Y. et al. Charge-transport in tin-iodide perovskite $\mathrm{CH}_{3} \mathrm{NH}_{3} \mathrm{SnI}_{3}$ : origin of high conductivity. Dalton T 40, 5563-5568 (2011).

34. Kontos, A. G. et al. Structural stability, vibrational properties, and photoluminescence in $\mathrm{CsSnI}_{3}$ perovskite upon the addition of $\mathrm{SnF}_{2}$. Inorg. Chem. 56, 84-91 (2017).

35. Ma, Y. C. et al. Controlled crystal facet of $\mathrm{MAPbI}_{3}$ perovskite for highly efficient and stable solar cell via nucleation modulation. Nanoscale 11, 170-177 (2019).

36. Chung, I., Lee, B., He, J. Q., Chang, R. P. H. \& Kanatzidis, M. G. All-solid-state dye-sensitized solar cells with high efficiency. Nature 485, 486-494 (2012)

37. Barlow, S. M., Bayatmokhtari, P. \& Gallon, T. E. $\mathrm{M}_{4,5} \mathrm{~N}_{4,5} \mathrm{~N}_{4,5}$ auger spectrum of tin and oxidized tin. J. Phys. C. Solid State 12, 5577-5584 (1979).

38. Kövér, L. et al. High-resolution photoemission and auger parameter studies of electronic-structure of tin oxides. J. Vac. Sci. Technol. A 13, 1382-1388 (1995)

39. Kövér, L. et al. Electronic structure of tin oxides: high-resolution study of XPS and auger-spectra. Surf. Interface Anal. 23, 461-466 (1995).

40. Lee, A. F. \& Lambert, R. M. Oxidation of Sn overlayers and the structure and stability of Sn oxide films on Pd(111). Phys. Rev. B 58, 4156-4165 (1998).

41. Fenwick, O. et al. Tuning the energetics and tailoring the optical properties of silver clusters confined in zeolites. Nat. Mater. 15, 1017-1022 (2016).

42. Satta, M. \& Moretti, G. Auger parameters and Wagner plots. J. Electron Spectrosc. 178, 123-127 (2010).

43. Asbury, D. A. \& Hoflund, G. B. A Surface study of the oxidation of polycrystalline tin. J. Vac. Sci. Technol. A 5, 1132-1135 (1987).

44. Cortecchia, D. et al. Lead-Free $\mathrm{MA}_{2} \mathrm{CuCl}_{\mathrm{x}} \mathrm{Br}_{4-\mathrm{x}}$ hybrid perovskites. Inorg. Chem. 55, 1044-1052 (2016).

45. Lu, N. D., Li, L. \& Liu, M. A review of carrier thermoelectric-transport theory in organic semiconductors. Phys. Chem. Chem. Phys. 18, 19503-19525 (2016)

46. Callaway, J. Model for lattice thermal conductivity at low temperatures. Phys Rev. 113, 1046-1051 (1959).

47. Pisoni, A. et al. Ultra-low thermal conductivity in organic-inorganic hybrid perovskite $\mathrm{CH}_{3} \mathrm{NH}_{3} \mathrm{PbI}_{3}$. J. Phys. Chem. Lett. 5, 2488-2492 (2014).

48. Linseis, V., Volklein, F., Reith, H., Nielsch, K. \& Woias, P. Advanced platform for the in-plane ZT measurement of thin films. Rev. Sci. Instrum. 89, 015110 (2018).

49. Burton, M. R. et al. Thin film tin selenide ( $\mathrm{SnSe})$ thermoelectric generators exhibiting ultralow thermal conductivity. Adv. Mater. 30, 1801357 (2018)

50. Volklein, F., Reith, H. \& Meier, A. Measuring methods for the investigation of in-plane and cross-plane thermal conductivity of thin films. Phys. Status Solidi A 210, 106-118 (2013).

\section{Acknowledgements}

The research was financed under O.F.'s Royal Society University Research Fellowship (UF140372). B.S. acknowledges financial support by the British Council (Grant No: 337323). T.L., X.Z., J.L. and Z.L. were supported by the Chinese Scholarship Council (CSC)

\section{Author contributions}

T.L. performed the experimental work on film deposition, structure and thermoelectric property characterization. X.Z. and J.L. performed the XRD measurements and data analysis. T.L., Z.L. and B.S. performed the XPS and AES measurements. F.L. and S.M. performed GIWAX measurements. This project was conceived and planned by O.F. and T.L and supervised by O.F. The paper was written with contributions from all authors

\section{Competing interests}

The authors declare no competing interests.

\section{Additional information}

Supplementary information is available for this paper at https://doi.org/10.1038/s41467 019-13773-3.

Correspondence and requests for materials should be addressed to O.F.

Peer review information Nature Communications thanks Peng Gao and the other anonymous, reviewer(s) for their contribution to the peer review of this work. Pee reviewer reports are available.

Reprints and permission information is available at http://www.nature.com/reprints

Publisher's note Springer Nature remains neutral with regard to jurisdictional claims in published maps and institutional affiliations. 
(c) (i) Open Access This article is licensed under a Creative Commons Attribution 4.0 International License, which permits use, sharing, adaptation, distribution and reproduction in any medium or format, as long as you give appropriate credit to the original author(s) and the source, provide a link to the Creative Commons license, and indicate if changes were made. The images or other third party material in this article are included in the article's Creative Commons license, unless indicated otherwise in a credit line to the material. If material is not included in the article's Creative Commons license and your intended use is not permitted by statutory regulation or exceeds the permitted use, you will need to obtain permission directly from the copyright holder. To view a copy of this license, visit http://creativecommons.org/ licenses/by/4.0/.

(C) The Author(s) 2019 